\title{
Commentary: Cannabinoids in Urology. Which Benign Conditions Might they be Appropriate to Treat: A Systematic Review \\ Charles Taylor $^{1 *}$, Brian Birch ${ }^{1,2}$ \\ University of Southampton, Faculty of Medicine \\ 2University Hospitals Southampton NHS FT, Southampton, UK
}

Article Info

\section{Article Notes}

Received: December 22, 2020

Accepted: January 15, 2021

\section{${ }^{*}$ Correspondence:}

${ }^{*}$ Dr. C Taylor, University Hospitals Southampton NHS FT, Southampton, UK; Email: ct1g17@soton.ac.uk.

${ }^{\circledR} 2021$ Charles Taylor. This article is distributed under the terms of the Creative Commons Attribution 4.0 International License.

\section{Introduction}

The recently published article in the Journal of Urology titled 'Cannabinoids in urology. Which benign conditions might they be appropriate to treat: A systematic review', explores the broad role of cannabinoids in the treatment of benign urological diseases. Whilst the review successfully appraises a significant amount of evidence, it was unable to discuss each urological application of cannabinoids in depth due to the current evidence base suffering from a distinct lack of in-depth analysis as well as due to manuscript word-limitations. The authors of the original review would like to use this commentary as an opportunity to further expand upon key elements that were not previously discussed.

The review states that cannabinoids and cannabis show most promise in the treatment of renal pathologies. However, an explanation as to why they show promise should also be provided and this is a chance to do so. It should be mentioned that modulation of the renal endocannabinoid system has been shown to be renoprotective in animal studies ${ }^{1}$. However, translational relevance to human studies is limited due to reports of acute kidney injury linked to synthetic cannabinoid use. Regardless, the review explains that dominant cannabinoid receptor type one (CB1) activation and cannabinoid receptor type two (CB2) inactivation results in renal oxidative stress, inflammation, cellular dysfunction, apoptosis and fibrosis of the kidneys. Equally, blocking CB1 receptors and stimulating CB2 receptors in the kidneys resets this imbalance in a reno-protective manner.

Whilst the review mentions the interplay between CB1 and CB2 receptors as a potential therapeutic strategy, this is a simplistic view as blocking one receptor and stimulating the other is challenging due to complex receptor interplay and hetero-dimerisation. It is also important to mention that CB1 receptors hetero-dimerise with angiotensin II type 1 receptors (AT1) ${ }^{2}$. In this respect, the relationship between the endocannabinoid system and reninangiotensin system requires further investigation.

The review provided a coherent overview of how cannabinoids and cannabis may be used within the context of renal pathologies however it did not mention that cannabinoids may be applicable to patients who have undergone renal transplantation for endstage chronic renal failure ${ }^{3,4}$. Chronic pain is a frequent issue in this patient group which is often due to underlying disease or intercurrent diseases. Transplant patients are restricted in their 
use of nonsteroidal anti-inflammatory drugs (NSAIDS) due to their nephrotoxic side effects. Whilst evidence is limited, cannabidiol (CBD) has displayed the potential to alleviate this pain, whilst being well tolerated with calcineurin inhibitors which are the foundation of immunosuppression for renal transplant patients ${ }^{3}$. Despite potential pharmacokinetic advantages, activation of the endocannabinoid system has been shown to down regulate the inflammatory response of macrophages and mesenchymal stromal cells and therefore may further contribute to the immunosuppression in this patient group $^{5}$. Regardless, opioids have long played a fundamental role in acute and chronic analgesia of such pain and cannabinoids could become an alternative analgesic option for this important patient group.

\section{Malignancy}

The review focuses on benign urological disease however the authors would like to use this commentary as a means of providing a brief overview of the applications of cannabinoids in malignant disease as this may potentially carry greater significance. Cannabinoids acting on both CB1 and CB2 receptors have been shown to either increase or decrease the risk of malignancy depending on the target organ ${ }^{6}$. Upon binding to CB receptors, cannabinoids can prevent cellular proliferation via cyclic adenosine monophosphate (cAMP)-dependent protein kinase A (pKA) inhibition which in turn prevents mitogen-activated protein kinase (MAPK) activation, leading to cell cycle arrest ${ }^{7,8}$. Such cannabinoid receptor activation also stimulates ceramide via serine pamitoyltransferase which induces $G_{1}$ cell cycle arrest and apoptosis ${ }^{7}$. Cannabinoids therefore clearly have a theoretical basis for the treatment of malignancy.

With further regards to malignancy, CB2 receptor activation leads to a decrease in the inflammatory cytokines, tumour necrosis factor alpha (TNF-alpha), Interleukin-6 (IL-6) and nuclear factor kappa light chain enhancer of activated B cells (NF-KB) as well as to the amelioration of reactive oxygen species (ROS) and reactive nitrogen species (RNS) ${ }^{9}$. CB2 agonists may therefore be used as antiinflammatory and antineoplastic agents whilst bypassing the central side effects associated with CB1 receptor activation. Whilst it is understood that the applications of cannabinoids in malignant disease were beyond the scope of the review, their significance and implications demand a detailed discussion.

\section{Pharmacokinetics and Pharmacodynamics}

The review identifies in its limitations that it does not discuss the side effects associated with cannabinoids and cannabis. This would have been explored in detail if script constraints had allowed. This is an important consideration for research regarding the potential pharmacological applications of cannabinoids.
The wide spectrum of side effects associated with cannabinoids are a result of activating cannabinoid receptors in a range of different tissues. Side effects include: short term memory impairment, impaired motor coordination, cognitive impairment and chronic bronchitis ${ }^{10}$. For each of these side effects there are varying degrees of evidence and support. Of note are psychological side effects which include an increased risk of schizophrenia, depression, anxiety, paranoia and reduced life satisfaction ${ }^{10}$. These dysphoric reactions are more common in naïve users, anxious subjects and psychologically vulnerable people and are greater with tetrahydrocannabinol (THC) compared to $\mathrm{CBD}^{11}$.

The pharmacokinetic profile of cannabinoids is largely unknown and the review did not address these points. If we are to act upon the conclusions of this review, then an understanding of the pharmacokinetics of cannabinoids and cannabis is required.

The most common routes of administration of cannabinoids are inhaled, oral, and oro-mucosal ${ }^{12,13}$. On inhalation, maximum plasma concentrations are reached after 3-10 minutes, psychotropic effects commence within seconds-minutes ${ }^{12,13}$. This is attributed to THC being rapidly transferred from the lungs to the blood. Once absorbed THC and other cannabinoids are distributed to other tissues at a rate dependent on blood flow ${ }^{11}$. By way of comparison an equal oral dose takes 2 hours to reach maximum plasma concentration due to continued slow absorption from the gut. If orally administered, psychotropic effects can last for up to 12 hours $^{11,14}$.

It is evident that cannabinoids have a complex and varied pharmacokinetic profile. Future research into cannabinoids as potential pharmaceutical agents would do well to explore this further so that we may gain a coherent understanding of their pharmacological behaviour.

\section{Summary and Implications}

The review correctly identified that not only is much of the evidence relating to cannabinoids and cannabis in a urological context conflicting, but there is also a lack of homogeneity between studies and a lack of data deemed scientifically rigorous enough to be translated to a clinical setting.

This could be in part due to the significant methodological challenges beyond those relating to social, legal and political issues. These include difficulty in standardisation of drug delivery and exposure as well as identification and control of cannabis strains.

It is important to emphasise that cannabinoids and cannabis may be classified as complementary therapies and, as is consistent with complementary therapies, their use in modern medicine has preceded scientific endorsement of their safety and efficacy. 
Before clinicians can utilise cannabinoids confidently as pharmaceutical agents, the biochemical mechanisms, pathophysiological pathways, relationships and interactions with the endocannabinoid system must be better elucidated and the appropriate clinical studies conducted.

This review successfully brings to attention the significant pharmacological potential of cannabinoids within urological and renal practice as well as the years of scientific neglect that cannabinoids have faced. Only following the implementation of future well designed and well sampled research might we see the translation of cannabinoids from recreational drugs to therapeutic agents. Currently, this is not possible.

\section{References}

1. Lecru L, Desterke C, Grassin-Delyle S, et al. Cannabinoid receptor 1 is a major mediator of renal fibrosis. 2015; 1(1): 72-84.

2. Hinden L, Tam J. Do Endocannabinoids Regulate Glucose Reabsorption in the Kidney? Nephron. 2019; 143(1): 24-7.

3. Cunetti L, Manzo L, Peyraube R, et al. Chronic Pain Treatment With Cannabidiol in Kidney Transplant Patients in Uruguay. Transplantation Proceedings. 2018; 50(2): 461-4.

4. Dao M, Lecru L, Vandermeersch S, et al. The cannabinoid receptor 1 is involved in renal fibrosis during chronic allograft dysfunction: Proof of concept. Journal of cellular and molecular medicine. 2019.
5. Ruhl T, Corsten C, Beier JP, et al. The immunosuppressive effect of the endocannabinoid system on the inflammatory phenotypes of macrophages and mesenchymal stromal cells: a comparative study. Pharmacological Reports. 2020: 1-11.

6. Rajanahally S, Raheem 0 , Rogers $\mathrm{M}$, et al. The relationship between cannabis and male infertility, sexual health, and neoplasm: a systematic review. Andrology.7(2): 139-47.

7. Sarfaraz S, Adhami VM, Syed DN, et al. Cannabinoids for cancer treatment: progress and promise. Cancer Res. 2008; 68(2): 339-42.

8. Zou S, Kumar U. Cannabinoid Receptors and the Endocannabinoid System: Signaling and Function in the Central Nervous System. Int. 2018; 19(3): 13.

9. Kumawat VS, Kaur G. Therapeutic potential of cannabinoid receptor 2 in the treatment of diabetes mellitus and its complications. European Journal of Pharmacology. 2019; 862: 172628.

10. Bridgeman MB, Abazia DT. Medicinal Cannabis: History, Pharmacology, And Implications for the Acute Care Setting. P T. 2017; 42(3): 180-8.

11. Ashton $\mathrm{CH}$. Pharmacology and effects of cannabis: a brief review. Br J Psychiatry. 2001; 178: 101-6.

12. Amin MR, Ali DW. Pharmacology of Medical Cannabis. Adv Exp Med Biol. 2019; 1162: 151-65.

13. Huestis MA. Human cannabinoid pharmacokinetics. Chem. 2007; 4(8): 1770-804.

14. Ho C, Martinusen D, Lo C. A Review of Cannabis in Chronic Kidney Disease Symptom Management. Canadian Journal of Kidney Health \& Disease. 2019; 6: 2054358119828391. 